\title{
Living in a LOFT
}

M. Feroci ${ }^{1,71}$, L. Stella ${ }^{2}$, M. van der Klis ${ }^{3}$, T. J.- L. Courvoisier ${ }^{4}$, M. Hernanz ${ }^{5}$, R. Hudec $^{6}$, A. Santangelo ${ }^{7}$, D. Walton ${ }^{8}$, A. Zdziarski ${ }^{9}$, D. Barret ${ }^{10}$, T. Belloni ${ }^{11}$, J. Braga $^{12}$, S. Brandt ${ }^{13}$, C. Budtz-Jørgensen ${ }^{13}$, S. Campana ${ }^{11}$, J.-W. den Herder ${ }^{14}$, J. Huovelin $^{15}$, G. L. Israel ${ }^{2}$, M. Pohl ${ }^{72}$, P. Ray ${ }^{16}$, A. Vacchi ${ }^{17}$, S. Zane ${ }^{8}$, A. Argan ${ }^{18}$, P. Attinà $^{19}$, G. Bertuccio ${ }^{20}$, E. Bozzo ${ }^{4}$, R. Campana ${ }^{1,71}$, D. Chakrabarty ${ }^{21}$, E. Costa ${ }^{1}$, A. De Rosa ${ }^{1}$, E. Del Monte ${ }^{1,71}$, S. Di Cosimo ${ }^{1}$, I. Donnarumma1, Y. Evangelista ${ }^{1}$, D. Haas $^{14}$, P. Jonker ${ }^{14}$, S. Korpela ${ }^{15}$, C. Labanti ${ }^{22}$, P. Malcovati ${ }^{23}$, R. Mignani ${ }^{8,24}$, F. Muleri $^{1}$, M. Rapisarda ${ }^{25,1,71}$, A. R. Rashevski ${ }^{17}$, N. Rea ${ }^{5}$, A. Rubini ${ }^{1,71}$, C. Tenzer ${ }^{7}$, C. Wilson-Hodge ${ }^{26}$, B. Winter ${ }^{8}$, K. Wood ${ }^{16}$, G. Zampa ${ }^{17}$, N. Zampa ${ }^{17}$, M. A. Abramowicz $^{27}$, M. A. Alpar ${ }^{28}$, D. Altamirano ${ }^{3}$, J. M. Alvarez ${ }^{5}$, L. Amati ${ }^{22}$, C. Amoros $^{10}$, L. A. Antonelli ${ }^{2}$, R. Artigue ${ }^{10}$, P. Azzarello ${ }^{4}$, M. Bachetti ${ }^{10}$, G. Baldazzi $^{29}$, M. Barbera ${ }^{30}$, C. Barbieri ${ }^{31}$, S. Basa ${ }^{32}$, A. Baykal ${ }^{33}$, R. Belmont ${ }^{10}$, L. Boirin ${ }^{34}$, V. Bonvicini $^{17}$, L. Burderi ${ }^{35}$, M. Bursa ${ }^{36}$, C. Cabanac $^{10}$, E. Cackett $^{37}$, G. A. Caliandro ${ }^{5}$, P. Casella ${ }^{38}$, S. Chaty ${ }^{46}$, J. Chenevez ${ }^{13}$, M. J. Coe $^{38}$, A. Collura ${ }^{30}$, A. Corongiu ${ }^{39}$, S. Covino $^{11}$, G. Cusumano ${ }^{40}$, F. D'Amico ${ }^{12}$, S. Dall'Osso ${ }^{41}$, D. De Martino ${ }^{42}$, G. De Paris $^{18}$, G. Di Persio ${ }^{1}$, T. Di Salvo ${ }^{30}$, C. Done ${ }^{43}$, M. Dovčiak ${ }^{36}$, A. Drago ${ }^{44}$, U. Ertan ${ }^{28}$, S. Fabiani ${ }^{1}$, M. Falanga ${ }^{45}$, R. Fender ${ }^{38}$, P. Ferrando ${ }^{46}$, D. Della Monica Ferreira ${ }^{13}$, G. Fraser $^{47}$, F. Frontera ${ }^{44}$, F. Fuschino ${ }^{22}$, J. L. Galvez ${ }^{5}$, P. Ghandi ${ }^{48}$, P. Giommi $^{49}$, O.

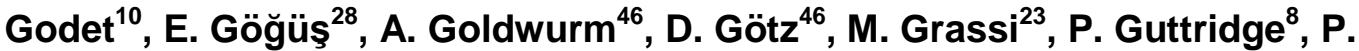
Hakala $^{50}$, G. Henri ${ }^{51}$, W. Hermsen ${ }^{14}$, J. Horak ${ }^{36}$, A. Hornstrup ${ }^{13}$, J.J.M. in 't Zand ${ }^{14}$, J. Isern ${ }^{5}$, E. Kalemci ${ }^{28}$, G. Kanbach ${ }^{52}$, V. Karas ${ }^{36}$, D. Kataria ${ }^{8}$, T. Kennedy ${ }^{8}$, D. Klochkov $^{7}$, W. Kluźniak ${ }^{9}$, K. Kokkotas ${ }^{7}$, I. Kreykenbohm ${ }^{53}$, J. Krolik ${ }^{54}$, L. Kuiper ${ }^{14}$, I. Kuvvetli ${ }^{13}$, N. Kylafis ${ }^{55}$, J.M. Lattimer ${ }^{56}$, F. Lazzarotto ${ }^{1}$, D. Leahy ${ }^{57}$, F. Lebrun ${ }^{46}$, D. $\operatorname{Lin}^{10}$, N. Lund ${ }^{13}$, T. Maccarone ${ }^{38}$, J. Malzac $^{10}$, M. Marisaldi $^{22}$, A. Martindale ${ }^{47}, M$. Mastropietro $^{1}$, J. McClintock ${ }^{58}$, I. McHardy ${ }^{38}$, M. Mendez ${ }^{59}$, S. Mereghetti ${ }^{60}$, M. C. Miller $^{61}$, T. Mineo ${ }^{40}$, E. Morelli ${ }^{1}$, S. Morsinck ${ }^{62}$, C. Motch ${ }^{34}$, S. Motta ${ }^{11}$, T. MuñozDarias $^{11}$, A. Naletto ${ }^{31}$, V. Neustroev ${ }^{63}$, J. Nevalainen ${ }^{15,50}$, J. F. Olive ${ }^{10}$, M. Orio ${ }^{64}$, M. Orlandini $^{22}$, P. Orleanski ${ }^{65}$, F. Ozel ${ }^{66}$, L. Pacciani ${ }^{1,71}$, S. Paltani ${ }^{4}$, I. Papadakis ${ }^{55}$, A. Papitto $^{39}$, A. Patruno ${ }^{3}$, A. Pellizzoni ${ }^{39}$, V. Petráček ${ }^{6}$, J. Petri ${ }^{34}$, P. O. Petrucci ${ }^{51}$, B. Phlips $^{16}$, L. Picolli ${ }^{23}$, A. Possenti ${ }^{39}$, D. Psaltis ${ }^{66}$, D. Rambaud ${ }^{10}$, P. Reig ${ }^{55,73}$, R. Remillard $^{21}$, J. Rodriguez ${ }^{46}$, P. Romano ${ }^{40}$, M. Romanova ${ }^{67}$, T. Schanz ${ }^{7}$, C. Schmid $^{53}$, A. Segreto ${ }^{40}$, A. Shearer ${ }^{68}$, A. Smith ${ }^{8}$, P. J. Smith ${ }^{8}$, P. Soffitta ${ }^{1}$, N. Stergioulas $^{69}$, M. Stolarski ${ }^{65}$, Z. Stuchlik ${ }^{70}$, A. Tiengo ${ }^{60}$, G. Török ${ }^{70}$, D. Torres ${ }^{5,74}$, R. 


\section{Turolla $^{31}$, P. Uttley ${ }^{38}$, S. Vaughan ${ }^{47}$, S. Vercellone ${ }^{40}$, R. Waters ${ }^{14}$, A. Watts ${ }^{3}$, R. Wawrzaszek $^{65}$, N. Webb ${ }^{10}$, J. Wilms ${ }^{53}$, L. Zampieri ${ }^{42}$, A. Zezas ${ }^{55}$, and J. Ziolkowski ${ }^{9}$}

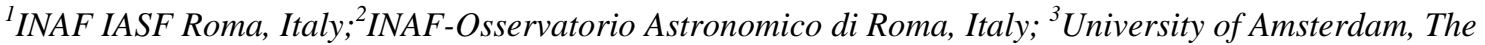
Netherlands; ${ }^{4} I S D C$, Geneve University, Switzerland; ${ }^{5}$ IEEC-CSIC, Spain; ${ }^{6}$ Czech Technical University, Czech Republic; ${ }^{7}$ Tuebingen University, Germany; ${ }^{8} \mathrm{MSSL}$, United Kingdom; ${ }^{9}$ N. Copernicus Astronomical Center, Poland; ${ }^{10}$ IRAP, France; ${ }^{11}$ INAF-OA Brera, Italy; ${ }^{12}$ INPE, Brazil; ${ }^{13}$ DTU Space, Denmark; ${ }^{14}$ SRON, The Netherlands; ${ }^{15}$ Helsinki University, Finland; ${ }^{16}$ NRL, Washington, USA $;{ }^{17}$ INFN, Trieste, Italy; ${ }^{18}$ INAF Headquarters, Rome, Italy; ${ }^{19}$ Thales-Alenia, Italy; ${ }^{20}$ Politecnico di Milano, Italy; ${ }^{21}$ MIT, USA; ${ }^{22}$ INAF-IASF-Bologna, Italy; ${ }^{23}$ Pavia University, Italy; ${ }^{24}$ Institute of Astronomy, University of Zielona G'ora Poland; ${ }^{25}$ ENEA Frascati,Italy; ${ }^{26}$ NASA/MSFC, USA; ${ }^{27}$ Goteborg University, Sweden; ${ }^{28}$ Sabanci University, Turkey; ${ }^{29}$ INFN, Bologna, Italy; ${ }^{30}$ Palermo University, Italy; ${ }^{31}$ Padova University, Italy; ${ }^{32}$ LAM - Laboratoire d'Astrophysique de Marseille, France; ${ }^{33}$ Technical University, Ankara, Turkey; ${ }^{34}$ Observatoire Astronomique de Strasbourg, France; ${ }^{35}$ Cagliari University, Italy; ${ }^{36}$ Prague Astron. Institute, Czech Republic; ${ }^{37}$ Cambridge University, United Kingdom; ${ }^{38}$ Southampton University, United Kingdom; ${ }^{39}$ INAF-OA Cagliari, Italy; ${ }^{40}$ INAF-IASF-Palermo, Italy; ${ }^{41}$ Racah Institute of Physics, Israel; ${ }^{42}$ INAF-OA Padova, Italy; ${ }^{43}$ Durham University, United Kingdom; ${ }^{44}$ Ferrara University, Italy; ${ }^{45}$ ISSI Bern, Switzerland; ${ }^{46}$ CEA Saclay, France; ${ }^{47}$ Leicester University, United Kingdom; ${ }^{48}$ SAS/JAXA, Japan; ${ }^{49}$ ASI, Italy; ${ }^{50}$ FINCA, Finnish Centre for Astronomy with ESO, Finland; ${ }^{51}$ Laboratoire Astrophysique de Grenoble, France; ${ }^{52}$ MPE, Germany; ${ }^{53}$ University of Erlangen-Nuremberg, Germany; ${ }^{54}$ Johns Hopkins University, USA; ${ }^{55}$ Crete University, Greece; ${ }^{56}$ State University of New York, USA; ${ }^{57}$ Calgary University, Canada; ${ }^{58}$ Harvard-Smithsonian Center for Astrophysics, United, States;

${ }^{59}$ Groningen University, The Netherlands; ${ }^{60}$ INAF-IASF-Milano, Italy; ${ }^{61}$ University of Maryland, USA; ${ }^{62}$ University of Alberta, Canada; ${ }^{63}$ Oulu University, Finland; ${ }^{64}$ INAF-OA Torino, Italy; ${ }^{65}$ Space Research Centre, Warsaw, Poland; ${ }^{66}$ University of Arizona, USA; ${ }^{67}$ Cornell University, USA; ${ }^{68}$ Galway University, Ireland; ${ }^{69}$ Thessaloniki University, Greece; ${ }^{70}$ Silesian University in Opava, Czech Republic; ${ }^{71}$ INFN Roma Tor Vergata Italy; ${ }^{72}$ DPNC, Geneva University, Switzerland; ${ }^{73}$ FORTH, Crete, Greece; ${ }^{74}$ ICREA, Spain.

LOFT (Large area Observatory For x-ray Timing) is an innovative mission concept for the next generation of X-ray experiments, submitted to the ESA Call for Medium size missions "M3". Recent developments in the field of Silicon detectors allowed us to design a realistic observatory devoted to X-ray timing studies with an effective area above $10 \mathrm{~m}^{2}$, operating in the energy range 2-30 keV with an energy resolution of $\sim 250 \mathrm{eV}$. Such an exceedingly large area (20 times that of RXTE/PCA), with a time resolution better than $10 \mu \mathrm{s}$, will enable unprecedently fast and accurate time variability studies related to accreting collapsed objects (e.g. fast coherent pulsations and QPOs). The scientific payload is complemented by a coded-mask wide field monitor based on similar detectors. In this paper we present the mission concept, the payload design and the expected performances.

Fast X-ray timing and spectroscopy at extreme count rates: Science with the HTRS on the International $X$-ray Observatory -HTRS 2011

Champéry, Switzerland

February 7-11 2011 


\section{Scientific motivation}

One of the topics of the European Space Agency (ESA) Cosmic Vision ${ }^{1}$ is the study of "Matter under extreme condition", related to the Theme 3, "What are the fundamental physical laws of the Universe?". In particular, the science topic is connected to the study of the General Relativity in the environment of black holes $(\mathrm{BH})$ and other compact objects and to the investigation of the equation of state (EOS) of matter in neutron stars (NS). These studies can be efficiently carried out with the high resolution timing and spectral observations of X-ray cosmic sources, exploiting the scientific performance of an innovative instrument such as LOFT.

The relation between mass and radius $(\mathrm{M}-\mathrm{R})$ in NS is a powerful probe of the EOS and allows to access regimes that cannot be studied in Earth-based laboratories. The two main techniques to investigate the EOS involve the modelling of the pulse profile in accreting millisecond pulsars (see for example Ref. [1]) and the observation of coherent oscillations during the rise phase of type I X-ray bursts (see Ref. [2]), when the thermonuclear emission from the hot spot is modulated by the NS spinning. Additional tools are represented by the derivation of $\mathrm{M}$ and $\mathrm{R}$ from the flux and temperature measurements during episodes of photospheric radius expansion of bright type I X-ray bursts from a source at known distance, as in globular cluster NS [3]. With its baseline $\sim 12 \mathrm{~m}^{2}$ effective area the Large Area Detector (LAD) aboard LOFT can reach an uncertainty smaller than 5\% (at 90\% confidence level) on the measurement of $\mathrm{M}$ and $\mathrm{R}$, and a comparable accuracy for the $\mathrm{M} / \mathrm{R}$ ratio from type $\mathrm{I}$ bursts. Constraints to the $\mathrm{M}-\mathrm{R}$ relation can be obtained also by the observation of QPOs and oscillations in flares from soft gamma-ray repeaters, a powerful tool in the study of the NS crust properties.

The motion of matter in the inner regions of the accretion disks around NS and down to the Innermost Stable Circular Orbit of BH, where most of the energy is emitted, is dominated by strong-field gravity effects. Two independent probes can be used for such measurements: the study of Quasi Periodic Oscillations (QPOs) in the flux of binary systems [4] and the analysis of the distortion of the Fe K $\alpha$ line profile emitted by accreting compact objects [5]. For the first time LOFT will carry out studies of individual pulses in low frequency QPOs and in kHz QPOs within their coherence timescale, enabling phase-resolved spectroscopy, especially in the spectral region around the $\mathrm{Fe}$ line. In the Active Galactic Nuclei (AGNs) the study of the variability of the relativistically broadened emission profile of $\mathrm{Fe} \mathrm{K} \alpha$ lines is facilitated by the higher rate per dynamical timescale, about $10^{6}$ times longer than in Galactic X-ray binaries. With a very high throughput, it will be possible to study the Fe line reverberation from the disk produced by individual blobs illuminated by the central source [6].

In addition to the science drivers outlined above, LOFT will exploit the physical information contained in the flux variability of X-ray sources, e. g. by detecting periodicities of extremely small amplitude and measuring their phase and period evolution with unprecedented accuracy. Since the X-ray sources are known to be extremely variable, the LOFT payload is completed by a coded aperture Wide Field Monitor (WFM, described in Sect. 2.2 below), able

\footnotetext{
${ }^{1}$ http://sci.esa.int/science-e/www/object/index.cfm?fobjectid=38657
} 
to monitor simultaneously large fractions of the sky with a sensitivity adequate to trigger LAD observations on outbursts and interesting states of known or previously undetected sources. Thanks to its large field of view and good angular resolution, the WFM will also be able to localise Gamma Ray Bursts, X-ray flashes, X-ray bursts and other fast transients.

\section{The LOFT mission concept}

The Large Observatory For $x$-ray Timing (LOFT) satellite-borne mission was designed to accomplish the scientific objectives outlined in Sect. 1 by fulfilling the scientific requirements summarised in Tab.1. The LOFT payload (see Fig. 1) is composed of two instruments: the LAD, a collimated instrument devoted to the timing and spectroscopy observation of sources in the soft X-ray band, and the WFM, a coded aperture and large field of view imager that will monitor the X-ray Sky in a similar energy band. Both LAD and WFM are based on the technology of large area linear Silicon Drift Detectors (SDDs), initially developed for charged particle tracking in High Energy Physics experiments (see Ref. [7]) and currently operating in the ALICE Inner Tracking system of LHC at CERN.

Tab. 1: Scientific requirements for the LOFT LAD and WFM.

\begin{tabular}{|l|l|l|l|}
\hline \hline LAD & Requirement & PFM & Requirement \\
\hline Parameter & & & \\
\hline \multirow{2}{*}{ Energy range } & $2-30 \mathrm{keV}$ nominal & Energy range & $2-50 \mathrm{keV}$ \\
\cline { 2 - 4 } & $2-50 \mathrm{keV}$ expanded & $\begin{array}{l}\text { Energy resolution } \\
\text { (FWHM at 6 keV) }\end{array}$ & $<300 \mathrm{eV}$ \\
\hline \multirow{2}{*}{ Effective area } & $12 \mathrm{~m}^{2}(2-10 \mathrm{keV})$ & Field of view $(\mathrm{FWHM})$ & $>3 \mathrm{sr}$ \\
\cline { 2 - 4 } & $1.3 \mathrm{~m}^{2}(30 \mathrm{keV})$ & Angular resolution & 5 arcmin \\
\hline \multirow{2}{*}{$\begin{array}{l}\text { anergy resolution }(\mathrm{FWHM} \text { keV) } \\
\text { Field of view (FWHM) }\end{array}$} & $<260 \mathrm{eV}$ & $\begin{array}{l}\text { Point source location } \\
\text { accuracy }\end{array}$ & 1 arcmin \\
\hline Time resolution & $<60 \mathrm{arcmin}$ & Sensitivity $(5 \sigma, 50 \mathrm{ks})$ & $2 \mathrm{mCrab}$ \\
\hline Dead time & $10 \mu \mathrm{s}$ & Sensitivity $(5 \sigma, 1 \mathrm{~s})$ & $0.5 \mathrm{Crab}$ \\
\hline Background flux & $<0.5 \%($ at $1 \mathrm{Crab})$ & & \\
\hline Max source flux (steady) & $>0.3 \mathrm{Crab}$ & & \\
\hline Max source flux (peak) & $>15 \mathrm{Crab}$ & & \\
\hline
\end{tabular}




\subsection{The Large Area Detector}

The LAD is a collimated instrument, reaching the unprecedented effective area of $\sim 12 \mathrm{~m}^{2}$, i. e. more than one order of magnitude larger than any similar instrument built in the past (e.g., RXTE/PCA, Ref. [8]). The innovative LAD design involves two key technologies, the Silicon Drift Detectors (SDDs) and the lead glass micro-capillary plates, enabling a very low mass per unit area. Thanks to the SDD technology, an energy resolution of $\sim 260 \mathrm{eV}$ FWHM at $6 \mathrm{keV}$ can be reached [9] and a bandpass of $\sim 2-50 \mathrm{keV}$. The LAD detector is highly segmented, thus making the dead time and pile-up unimportant for most of the

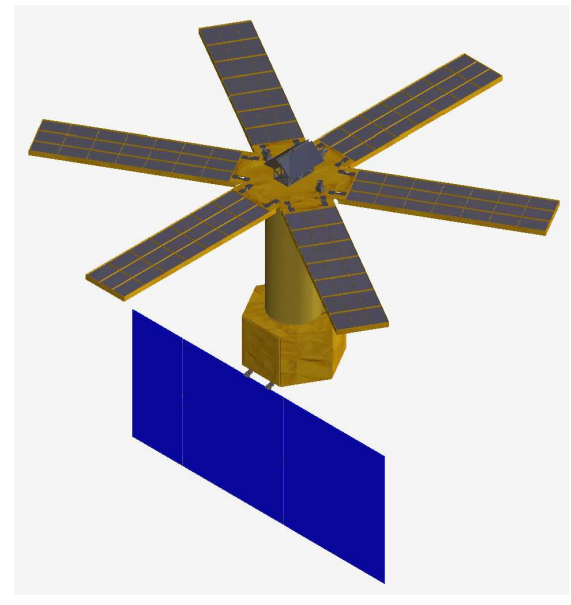

Figure 1: the LOFT payload observations. The LAD adopts a modular approach, using about 2000 SDDs, each one $450 \mu \mathrm{m}$ thick and with $\sim 76 \mathrm{~cm}^{2}$ geometric area, read-out in one-dimension every $\sim 850 \mu \mathrm{m}$. The LAD collimator is based on the same technology as the microchannel plates. An array of multipore lead glass slabs, with thickness of $\sim 2 \mathrm{~mm}$, inner hole diameter of $\sim 25 \mu \mathrm{m}$ and pitch of $\sim 28 \mu \mathrm{m}$, is used to absorb X-rays coming from outside the $\sim 40$ arc min aperture up to $\sim 50 \mathrm{keV}$. An increasing transparency of the collimator at energies above $\sim 30 \mathrm{keV}$ offers a response to offaxis hard and bright events (e.g., gamma ray bursts or magnetar flares) at the price of a slight increase in the background. A key feature of this design for the collimator is its very low weight, approximately $3 \mathrm{~kg} / \mathrm{m}^{2}$, enabling a large area at a reasonable mass.

The modular design of the LAD allows the use of a standard service module ${ }^{2}$ for LOFT, that can be efficiently stowed inside the launcher fairing volume of Vega, the small ESA launcher. In fact, the LAD is composed of six panels, initially folded and deployed after the launch. A tight requirement on the alignment of the six panels can be fulfilled using a deployment technology ${ }^{2}$ derived from the antenna of the Synthetic Aperture Radar systems, used e. g. in the MIRAS payload of the ESA SMOS mission [10].

\subsection{The Wide Field Monitor}

The WFM is in charge of monitoring the flux and spectral variability of the X-ray sources within the sky fraction accessible to the LAD, triggering its observations on interesting states and providing the context of the pointed LAD observations. The WFM is a one-dimensional coded aperture experiment based on linear SDDs, similar to those adopted by the LAD, but with a smaller pitch, $\sim 300 \mu \mathrm{m}$. The SDDs allow to obtain an energy resolution of $\sim 3-400 \mathrm{eV}$ over an energy range of 2-50 keV.

A total of 4 cameras are currently envisaged for the WFM, co-aligned in pairs to achieve a combined 2D imaging, similar to previous experiments (e.g., RXTE/ASM, HETE-2/WXM or SuperAGILE). A rough $2 \mathrm{D}$ encoding is indeed available also by each individual camera by

${ }^{2}$ Other images and sketches of the LOFT payload, the deployment system, the LAD and WFM can be found on the LOFT website, http://www.isdc.unige.ch/loft/index.php/instruments-on-board-loft 
exploiting the drift properties of the detectors [e.g., 11]. Combining this feature with a $\sim 100 \mu \mathrm{m}$ thick Tungsten coded mask at $\sim 15 \mathrm{~cm}$ distance, the WFM will achieve an angular resolution of 5 arcmin $x$ few degrees for each of the 4 cameras. The simultaneous observation of the same sources with two co-aligned but orthogonal cameras will enable a point source location accuracy of about 1 arcmin in two-dimensions, for sources brighter than a few mCrab (1-day integration).

\subsection{Mission profile}

Although the LAD observations of Galactic sources are expected to be largely sourcedominated, the scientific objectives related to the observation of AGNs are sensitive to the background minimization and stability, thus a LEO orbit with $<600 \mathrm{~km}$ altitude and $<5^{\circ}$ inclination was selected. In the preliminary baseline the LOFT spacecraft has a fixed solar panel array with $\pm 20^{\circ}$ aspect angle constraints and will operate in three-axis stabilized pointing mode, with a slew rate capability of $4 \%$ minute. LOFT has a higher data throughput with respect to previous missions, thus we envisage a telemetry down-link to two ground stations, Kourou (ESA) and Malindi (ASI), with a total data rate of $\sim 700 \mathrm{kbit} / \mathrm{s}$ (orbit average). In this scenario each ground station will have a $\sim 10$-min contact every $\sim 100$-min orbit. Kourou will be used for both telecommand up-link and telemetry down-link, while Malindi for telemetry down-link only. Additional details on the design and performance of LOFT may be found in Ref. [12].

\section{References}

[1] D. A. Leahy et al., ApJ, 691, 1235 (2009)

[2] M. P. Muno et al., ApJ, 542, 1016 (2000)

[3] A. W. Steiner t al., ApJ, 722, 33 (2010)

[4] M. van der Klis, $A R A \& A, \mathbf{3 8}, 717$ (2000)

[5] A. Fabian et al., MNRAS, 238, 729 (1989)

[6] M. Dovciak et al., MNRAS, 350, 745 (2004)

[7] E. Gatti \& P. Rehak, Nucl. Instr. and Meth. A, 225, 608 (1984)

[8] K. Jahoda et al., ApJSS, 163, 401 (2006)

[9] G. Zampa et al., Nucl. Instr. and Meth. A, 633, 15 (2011)

[10] J.I. Bueno et al., ESA-SP 591235 (2005)

[11] R. Campana et al., Nucl. Instr. and Meth. A, 633, 22 (2011)

[12] M. Feroci et al., Exp. Astr., in press (2011), [arXiv:1107.0436] 